Studie über Kleintransporte und Dienstleistungsverkehr

\title{
Modellhafte Maßnahmen
}

\section{Ein Forschungsprojekt zur Erprobung von Maßnahmen zur umweltschonenden Abwicklung des städtischen Wirtschaftsverkehrs am Institut für ökologische Wirtschaftsforschung widmet sich vor allem den Personenwirtschaftsver- kehren, die nicht ausdrücklich der Güferbeförderung dienen sowie Kleintrans- porten, die mit Fahrzeugen bis zu 3,5 Tonnen zulässigem Gesamtgewicht durchgeführt werden.}

I Von Wulf-Holger Arndt m Rahmen des Umweliforschungsplans 1996 des Umweltbundesamtes hat das IÖW federfiihrend die Vorstudie „Erprobung von Maßnahmen zur umweltschonenden Abwicklung des städtischen Wirtschaftsverkehrs" bearbeitet. Auf der Basis einer qualitativen und soweit möglich quantitativen Differenzierung und Beschreibung des Untersuchungsgegenstandes wurden modellhafte Maßnahmen zur Gestaltung des Wirtschaftsverkehrs entwickelt, die sich wesentlich auf den Handlungsrahmen der kommunalen Planung unter Mitwirkung der Unternehmen beziehen. In diesem Vorhaben geht es explizit um zwei in der Planung bisher wenig berücksichtigte Verkehrsarten: Zum einen um Personenwirtschaftsverkehre, die nicht ausdrücklich der Güterbeförderung dienen (Dienstleistungsfahrten z. B. von Handwerkern, Handelsvertretern, Kurier- und Expressdiensten) und überwiegend mit Pkw und Kombi erfolgen; sowie um Kleintransporte, die mit Fahrzeugen bis zu 3,5 Tonnen zulässigem Gesamtgewicht durchgeführt werden.

Mittlerweile wird an der Vorbereitung der praktischen Umsetzung modellhafter Maßnahmen in Kommunen gearbeitet, wobei als Modellstädte Augsburg, Hamm und Berlin ausgewählt wurden. Das Maßnahmenfeld, das in Augsburg erprobt wird, ist die planerische Flankierung umweltfreundlicher Fahrzeugtechnik. Da sich die Stadt Augsburg um die Teilnahme am Investitionsvorhaben ,Modellhafter Einsatz von gasbetriebenen Fahrzeugen" des BMU/UBA beworben hat, werden die Projekte „Modellhafter Einsatz gasbetriebener Fahrzeuge" und ,Erprobung von Maßnahmen zur umweltschonenden Abwicklung im städtischen Wirtschaftsverkehr" miteinander verknüpft.

Das IÖW ist beauftragt, bei folgenden Aufgaben im Investitionsvorhaben eine wissenschaftliche Begleitung durchzufuihren:
- Auswahl geeigneter Betriebe (Handwerker, Dienstleister) für den praktischen Einsatz von Gasfahrzeugen,

- gemeinsame Erarbeitung von planerischen Maßnahmen (Benutzervorteile beim Einsatz von Gasfahrzeugen, Straßenraumgestaltung, Parkraummanagement),

- vergleichende Erhebung und Bewertung von Transportketten (mit und ohne umweltentlastende Maßnahmen),

- Umsetzung der verkehrsplanerischen Maßnahmen.

Für die Vermarktung der Fahrzeugförderung hat der Hauptauftragnehmer, die Stadtwerke Augsburg, eine Marketingfirma beauftragt. Am 1. Juli wurden die ersten Förderbescheide im Rahmen einer öffentlichen Veranstaltung auf dem Marktplatz der Stadt durch die Umweltbundesministerin Merkel an Augsburger Unternehmen übergeben. Die Stadtwerke selbst sind zur Zeit das Unternehmen, das die meisten Gasfahrzeuge einsetzt. Gasbusse prägen inzwischen das Bild der Buslinien im öffentlichen Stadtverkehr. Die großflächigen Aufkleber ,Gasantrieb - umweltschonend, weil lärmarm und besonders schadstoffarmer Antrieb" sind aber auch auf anderen Fahrzeugen im Stadtbild sichtbar. Insgesamt sind bis jetzt circa 60 Fahrzeuge in Augsburg durch Mittel des Vorhabens gefördert worden. Für 52 weitere Gasfahrzeuge liegen Anträge auf Förderung vor. In den Förderanträgen privater Unternehmen werden überwiegend Pkw und Transporter nachgefragt. Das Branchenspektrum reicht vom Elektrohandwerker und Reinigungsunternehmen über die Spedition und den Taxibetrieb bis zum Notarzt und Seniorenheim. Zahlreiche Fahrzeughersteller bieten z. Zt. gasbetriebene Fahrzeuge oder Umrüstungssätze für Pkw oder Nutzfahrzeuge an. Die im Vergleich zu konventionellen Fahrzeugen momentan höher liegenden Anschaffungskosten oder die Zusatzko- sten für die Umrüstung werden bis zu 80 Prozent durch das Umweltbundesamt ausgeglichen. Durch die Senkung der Mineralölsteuer für den Kraftstoff Erdgas ergibt sich jedoch für die Betreiber solcher Fahrzeuge ein finanzieller Vorteil gegeniber Diesel- oder Benzinfahrzeugen. Aufgrund des geringen Schadstoffausstosses erfiillen diese Fahrzeuge heute bereits die ab 1999 gilltige Euro3-Norm. Eine Angebotsliicke besteht allerdings noch bei Lkw mit vier bis acht Tonnen Nutzmasse. Ebenfalls hinderlich für die Durchsetzung dieser umweltschonenden Fahrzeugtechnik ist die geringe Dichte des Gastankstellennetzes. Besonders bei Überlandfahrten sind ohne größere Umwegfahrten oft die Tankstellen nicht erreichbar. Die Fuhrunternehmen müssen darauf mit stärkerer Optimierung bei der Routenplanung bis hin zur Beschränkung des Einsatzes auf das Stadtgebiet reagieren. Die Einsatzflexibilität der Fahrzeuge ist somit eingeschränkt. Verbesserungen zeichnen sich allerdings für die Zukunft ab. Bundesweit befinden sich 55 und in Augsburg eine Gastankstelle in Betrieb. In der Stadt sind weitere drei, und bundesweit ca. 30 Standorte in Planung.

Wichtig für die Akzeptanz der neuartigen Fahrzeuge bei den Transportunternehmen ist die Einräumung von Benutzervorteilen. Bestandteil des Vorhabens ist ein Rechtsgutachten zur Prüfung von solchen Vorteilen. Neben den Gasfahrzeugen werden im Gutachten auch solche Fahrzeuggruppen betrachtet, die durch ihre Beteiligung an einem Poolsystem zur überbetrieblichen Auslastungs- und Fahrtenoptimierung (City-Logistik) $\mathrm{zu}$ einer wesentlichen Verringerung des Verkehrsaufwandes und der Emissionen beitragen. Dabei werden folgende Punkte untersucht:

- die Kennzeichnungsmöglichkeit der Fahrzeuge, - die bevorzugte Nutzung von Parkplätzen und Ladezonen,

- die Bevorzugung bei Ausnahmegenehmigungen bei Fahrverboten oder -einschränkungen in bestimmten Straßen oder zu bestimmten Zeiten, - Aussetzen von Park- und Haltebeschränkungen und

- die Einräumung von Ausnahmen von Geschwindigkeitsbeschränkungen.

Im Vorfeld kam es hinsichtlich des Kennzeichentextes $\mathrm{zu}$ einem juristischen Verfahren zwischen der Stadt Augsburg und dem Mineralölwirtschaftsverband. Die Formulierung „umweltfreundlich" ist angreifbar. Inzwischen konnte bereits eine rechtssichere Kennzeichnung für die Fahrzeuge erarbeitet werden. Die 


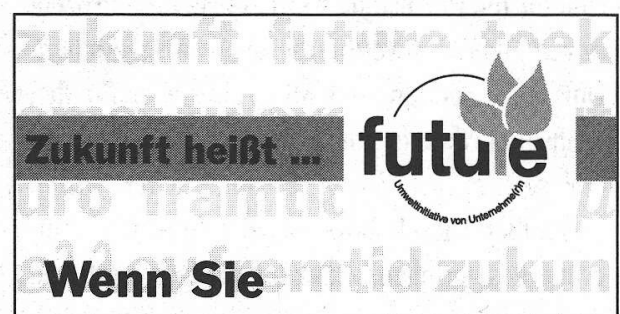

verantwortungsbewuBt wirtschaften wollen ...

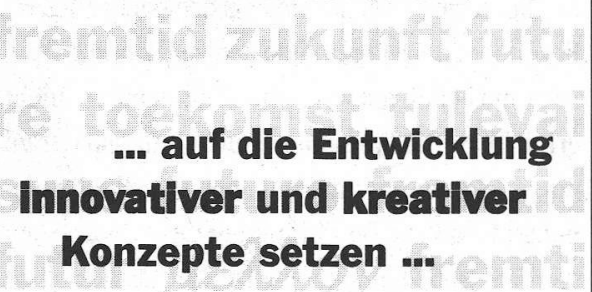

\section{... Okologisch und wirtschaftlich handeln müssen ...}

\section{... eine umweltorientierte} und sozlal verpilichtete Marktwirtschaft gestalten möchten ...

... europallsch denken können ...

\section{dann machen Sie mit uns}

\section{die Zukunft zur Chance!}

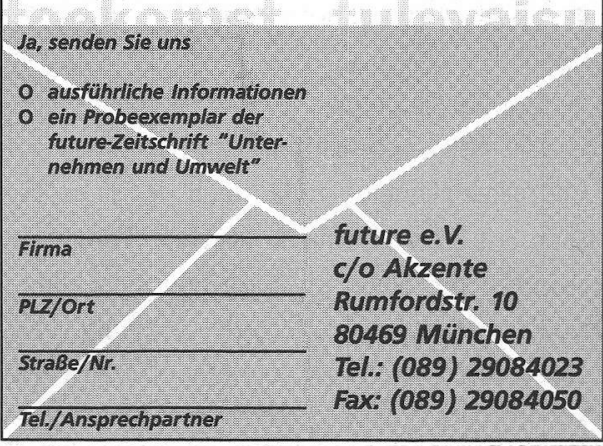

Aufschrift auf den Fahrzeugen sollte ,Gasmotor - umweltschonend, weil lärmarm und besonders schadstoffarmer Antrieb" lauten. Die jetzigen Arbeiten zum Rechtsgutachten konzentrieren sich auf die wettbewerbsrechtlichen, straßenrechtlichen und straßenverkehrsrechtlichen Fragen zu den Benutzervorteilen. Mit der Stadt wird gegenwärtig die Umsetzung möglicher Benutzervorteile besprochen.

Für die Untersuchung der Einsatzerfahrungen bezüglich der Gasfahrzeuge ist eine Befragung unter den Betreibern vorgesehen. Neben technischen Aspekten werden auch Kennwerte zum betrieblichen Einsatz erfaßt. Von Interesse ist dabei (neben den Erfahrungen zur technischen Zuverlässigkeit der Fahrzeuge), wie die Unternehmen die Gasfahrzeuge einsetzen. Gibt es Unterschiede zu den Einsatzprofilen der herkömmlichen Fahrzeuge? Welche Rolle spielt die bereits angesprochene Problematik der Tankstellendichte? Welche Wirkungen haben die eingeräumten Benutzervorteile auf die Fahrzeugplanung der Fuhrunternehmen?

Die Wirkung des Einsatzes der umweltschonenden Fahrzeuge auf die Schadstoffbilanz wird anhand von Vorher- bzw. Nachher-Vergleichen vom IÖW erarbeitet. Mit Hilfe des Programmpakets MOBILEV ist eine Simulation der Emissionen in Straßen möglich.

Eine Auflage für die Teilnahme am Investitionsvorhaben war die Durchfiuhrung von flankierenden verkehrsplanerischen Maßnahmen. In Augsburg wurden und werden dazu eine ganze Reihe von Maßnahmen umgesetzt. Ziel dieser Bemühungen ist die umweltgerechtere Gestaltung des Verkehrssystems. Als gelungenes Beispiel kann das Busbeschleunigungskonzept für den öffentlichen Personennahverkehr (ÖPNV) genannt werden. Bis jetzt sind 160 lichtsignalgesteuerte Straßenkreuzungen mit Vorrangschaltungen für den ÖPNV versehen. Die Standzeiten vor den Ampeln haben sich für Busse und Bahnen in den meisten Fällen auf ein Minimum verringert. Der Ausbau des Straßenbahnnetzes wird auch gegen Widerstände von seiten der Autolobby weiter vorangetrieben. Das Planfeststellungsverfahren für die Verlängerung der Linie 2 ist vor kurzem fertiggestellt worden. Andererseits konnte der geplante Bau einer innenstadtnahen vierspurigen Straße nicht verhindert werden. $\mathrm{Zu}$ diesem Projekt fand eine öffentliche Auseinandersetzung statt, die schließlich in einen Volksentscheid mündete.

Die Stadt Augsburg sieht in der Attraktivitätsstei- gerung des Eisenbahnverkehrs ebenfalls ein wichtiges Element für die umweltgerechtere Verkehrsgestaltung. Neben Verbesserungen für die Regionalbahn wird auch ein Spezifikum im Augsburger Schienennetz, die Localbahn, in die Überlegungen miteinbezogen. Da die Localbahn über eine Meterspur verfügt, ist eine Verknüpfung mit der Straßenbahn gleicher Spurbreite denkbar. Durch ihr Streckennetz, das fast die gesamte Innenstadt umschließt und Anschlußstrecken in die größeren Gewerbegebiete besitzt, sollen Straßen von Lkw-Verkehr entlastet werden. Für den verbleibenden Lkw-Verkehr wird ein Wegweisungssystem installiert, das die Umfahrung sensibler Stadtbereiche erleichtert. Die Einrichtung einer City-Logistik-Gemeinschaft zielt neben den wirtschaftlichen Aspekten auf die grundsätzliche Verringerung von LkwFahrten. Neben den sechs Gründungsmitgliedern haben sich allerdings keine weiteren Speditionen angeschlossen. Die Vorteile einer solchen Zusammenarbeit fuir Stadt und Wirtschaft sollen durch eine Güterverkehrsrunde stärker in der Öffentlichkeit bekanntgemacht werden.

Ein wichtiger Aspekt bei der Verkehrsvermeidung ist die Gestaltung von verkehrsminimierenden Siedlungsstrukturen. Bei der Verwirklichung von Wohngebieten mit verkehrsvermeidenden Raumstrukturen hat die Stadt unterschiedliche Erfahrungen zu verzeichnen. Beim Projekt im PrinzKarl-Viertel kann nach Kontakt zur obersten Baubehörde jetzt jeder Investor über die Entwicklung seiner Flächen zu autoarmen Wohngebieten entscheiden. Durchgangsverkehr ist für dieses Gebiet ausgeschlossen. Investoren für die Schaffung von wohnortnahen und umweltverträglichen Arbeitsplätzen sind gefunden worden. Eine Mobilitätszentrale für dieses Gebiet ist geplant. Ein anderes Gebiet, Haunstetten Süd, hat zur Zeit keine Realisierungschancen, da der Markt als gesättigt angesehen wird.

\section{Anmerkungen}

(1) An dieser Vorstudie waren außer dem löW das Planungsbüro Junker+Kruse, Dortmund, die IVU GmbH, Berlin, sowie Dr. Schütte, Universitüt Dortmund beteiligt (vgl. dazU auch die IÖW-Schriftenreihe 107/97 von Dr. Schütte).

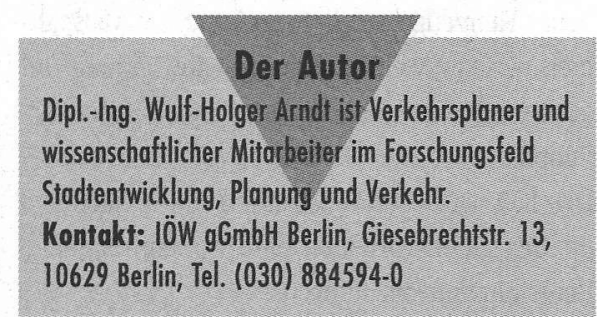


(c) 20I0 Authors; licensee IÖW and oekom verlag. This is an article distributed under the terms of the Creative Commons Attribution Non-Commercial No Derivates License (http://creativecommons.org/licenses/by-nc-nd/3.o/), which permits unrestricted use, distribution, and reproduction in any medium, provided the original work is properly cited. 